\title{
In patients with head injury undergoing rapid sequence intubation, does pretreatment with intravenous lignocaine/lidocaine lead to an improved neurological outcome? A review of the literature
}

\author{
N Robinson, M Clancy
}

\begin{abstract}
It is well known that laryngeal instrumentation and endotracheal intubation is associated with a marked, transient rise in intracranial pressure (ICP). Patients with head injury requiring endotracheal intubation are considered particularly at risk from this transient rise in ICP as it reduces cerebral perfusion and thus may increase secondary brain injury. The favoured method for securing a definitive airway in this patient group is by rapid sequence intubation (RSI). In the United States the Emergency Airway Course teaches emergency physicians to routinely administer intravenous lidocaine as a pre treatment for RSI in this patient group in an attempt to attenuate this rise in ICP. A literature search was carried out to identify studies in which intravenous lidocaine was used as a pretreatment for RSI in major head injury. Any link to an improved neurological outcome was also sought. Papers identified were appraised in the manner recommended by the evidence based medicine group to ensure validity. There were no studies identified that answered our question directly and, furthermore, it is our belief that no such study, at present, exists in the literature. Six valid papers were found, which individually contained elements of the question posed and these are presented in a narrative and graphic form. There is currently no evidence to support the use of intravenous lidocaine as a pretreatment for RSI in patients with head injury and its use should only occur in clinical trials. (Emerg Med F 2001;18:453-457)
\end{abstract}

Keywords: head injury; rapid sequence intubation; lidocaine; lignocaine

Emergency

Department,

Southampton General

Hospital, Tremona

Road, Southampton

SO16 6YD, UK

Correspondence to: Dr Robinson

(poppabear66@hotmail.com)

Accepted for publication 21 July 2001
Advanced airway management is of critical importance in the resuscitation of patients with head injuries with a depressed level of consciousness. Rapid sequence intubation (RSI) refers to the virtual simultaneous administration of a potent sedative agent and a neuromuscular blocking agent to induce unconsciousness and motor paralysis for the purpose of facilitating endotracheal intubation. The placement of a definitive airway, in the patient with head injury, offers several advantages, namely that the patient is protected from inadvertent aspiration of gastric contents, accurate oxygen delivery is maintained and, by controlling ventilation, hypercapnia can be avoided.

It has been shown in numerous studies that instrumentation of the larynx and the placement of an endotracheal tube is associated with a marked, transient increase in both mean arterial pressure and heart rate. ${ }^{1-4}$ This has been termed the "pressor response" and is attributable to the sudden release of plasma catecholamines occurring as a consequence of laryngoscopy and endotracheal intubation..$^{4-8}$ In the patient with head injury the pressor response to intubation may contribute to secondary brain injury in the following ways by:

(1) Causing a sudden increase in cerebral blood volume raising intracranial pressure (ICP).

(2) Increasing cerebral oedema formation because of the sudden rise in capillary hydrostatic pressure.

(3) Increasing haemorrhage and haematoma formation.

(4) Causing systemic and cerebral venoconstriction to occur raising central venous pressure (CVP).

In the emergency management of major head injuries all efforts are directed at the maintenance of adequate cerebral perfusion pressure (CPP), maximising tissue oxygenation and the control of ICP in order to minimise secondary brain injury. The CPP is dependent on the mean arterial pressure, the ICP and the CVP. Any rise in ICP or CVP directly reduces the CPP and so increase ischaemia in the injured brain. It has also been shown that ICP rises during laryngeal instrumentation and endotracheal intubation in patients undergoing elective neurosurgery, for cerebral neoplasia or aneurysms, and in patients with brain injury undergoing endotracheal suctioning. ${ }^{9}{ }^{10}$ The precise mechanism by which endotracheal intubation and suctioning stimulates a rise in ICP is not well elucidated. It may be as a consequence of the pressor response, as described above. However, one study has postulated that a separate centrally mediated neuronal pathway, possibly linked to the cough reflex, may also be responsible. ${ }^{11}$

In the past it was recommended that intravenous lignocaine/lidocaine should be 
Table 1

\begin{tabular}{llll}
\hline Search engine & Papers identified & $\begin{array}{l}\text { Number directly } \\
\text { relevant to question }\end{array}$ & $\begin{array}{l}\text { Number included in the } \\
\text { review (see results for } \\
\text { explanation) }\end{array}$ \\
\hline Medline & 5 & 0 & 1 \\
EMBASE & 4 & 0 & 1 \\
PubMed & 9 & 0 & 2 \\
Bibliographies & 8 & 0 & 2 \\
\hline
\end{tabular}

given to attenuate the pressor response to laryngeal instrumentation and endotracheal intubation but the results of studies done on this subject have been contradictory and inconclusive. ${ }^{12-20}$ A survey of Emergency Medicine Residency Programs in the United States (US) revealed that intravenous lignocaine/ lidocaine was routinely administered prior to RSI in patients with major head injuries to attenuate any rise in ICP associated with laryngeal instrumentation and endotracheal intubation, ${ }^{21}$ However, a recent survey of RSI practice in English emergency departments demonstrated the complete absence of such pretreatment. ${ }^{22}$ Such disparity between US and English practice prompted this review.

\section{Method}

Using the three part question recommended by Sackett, ${ }^{23}$ our question is:

In patients suffering a major head injury who undergo RSI, does pretreatment with intravenous lignocaine/lidocaine compared with no pretreatment lead to an improved neurological outcome?

Relevant literature was identified in the following ways:

(1) Electronic database searches. Medline (1966 to present), EMBASE (1988 to present), PubMed search using Doctors net UK and the Cochrane Library. The searches were limited to human studies published in any language. The title and abstract (if available) was read and if relevant or considered potentially relevant the full paper was obtained and appraised and the level of evidence graded (see text).

\section{TERMS USED}

(i) [ $\{\exp$ lidocaine OR lignocaine.mp OR lidocaine.mp\} AND \{exp intubation, intratracheal OR rapid sequence intubation \$.mp OR exp endotracheal intubation OR endotracheal intubation $\$ . m p$ AND exp intracranial pressure OR intracranial pressure $\$ . m p$ OR ICP $\$ . m p$ \} AND exp craniocerebral trauma $O R$ exp brain injury OR head injury $\$ . m p$ OR traumatic coma\$.mp\}] LIMIT to human and english language.

The advanced search using Doctors Net UK asks for search terms such as "Rapid sequence intubation and lidocaine and intracranial pressure" then allows selection of "articles related to therapy" and either "sensitive" or "specific" filters. The papers identified have a "related articles" icon which, when accessed, networks the reader to many other papers that may, or may not be of relevance. Any article of relevance found here, itself has a "related articles" icon that can be further searched increas- ing the sensitivity of any search done. This lead to the scanning of 500 titles.

(2) The bibliographies of the papers obtained were searched and any relevant or possibly relevant papers were obtained and appraised.

(3) Advice from experts in the field was sought.

Those prospective, randomised, controlled trials (PRCTs) identified were appraised in the manner recommended by the evidence based medicine group using the following criteria:

THE MAIN QUESTIONS TO ANSWER:

(a) Was the assignment of patients to treatments randomised and was the randomisation list concealed?

(b) Were all patients who entered the trial accounted for at its conclusion?

(c) Were they analysed in the groups to which they were randomised?

THE LESSER QUESTIONS TO ANSWER:

(d) Were patients and clinicians kept blind to which treatment was being received?

(e) Aside from the experimental treatment, were the groups treated equally?

(f) Were the groups similar at the start of the trial?

Relevant observational studies were also included if the information they contained was directly related to the questions posed.

In order to describe the "fit" of the literature to our question the following approach was used. Each of the three parts of the question were represented graphically along three different axes. The fourth axis represented the level of evidence (see later). Each axis is considered equally important. An ideal paper-that is, one that exactly matched each of the three parts of the question, and was a systematic review or RCT with narrow confidence intervals - would be positioned at the origin of the axes. The points along scale of each axis are derived from the literature review (see later) and represent decrements in "fit". Thus the further from the origin the less exact the match of that study's attributes to the ideal. For the purposes of this paper and to facilitate interpretation by the reader, the difference between each point along the axis, although of a qualitative nature, are treated as equal. That is, these ordinal measurements were treated as interval measurements.

\section{Results}

The results of the search are summarised in table 1.

It was clear from the search that no evidence exists that answers our specified question. Using each of the three components of the question and the level of evidence the grid was constructed to illustrate how close the papers came to answering the question (fig 1).

The patient population $(P)$ was those patients presenting to the emergency department with acute traumatic brain injury $(<12$ hours from the time of injury) requiring RSI as a component of their emergency management. No such study was identified. The closest 
reported group were comatose patients suffering from closed traumatic head injury ventilated on intensive care units, already intubated. The other reported group were patients undergoing elective neurosurgery for tumours or intracranial aneurysms with or without preexisting raised ICP. For the purposes of this paper, along an interval scale patients suffering acute traumatic brain injury were scored 0 , patients with head injury treated on intensive care units 1, and those undergoing elective neurosurgery for brain tumours or aneurysms 2.

The Intervention(I) was pretreatment with intravenous lignocaine/lidocaine in those patients with head injury undergoing RSI. No study dealt with RSI but some described elective anaesthesia and intubation while others evaluated the effect of lignocaine/lidocaine administered intravenously prior to endotracheal suctioning. Patients undergoing RSI were scored 0 , elective anaesthesia and intubation 1 , and patients anaesthetised and undergoing endotracheal suctioning but not undergoing the act of intubation, 2 .

The ideal outcome $(O)$ would be their neurological status at discharge or later. No paper reported this outcome and the surrogate marker of intracranial pressure change was used. Patients with neurological status at discharge were scored 0 , those whose intracranial pressure was measured 1 , and those whose spinal pressure was measured 2 .

Sackett et al have described levels of eviden$c e(L)$ for a treatment as follows:

1a Systematic review of randomised controlled trials (RCT)

1b Individual RCT with narrow confidence intervals
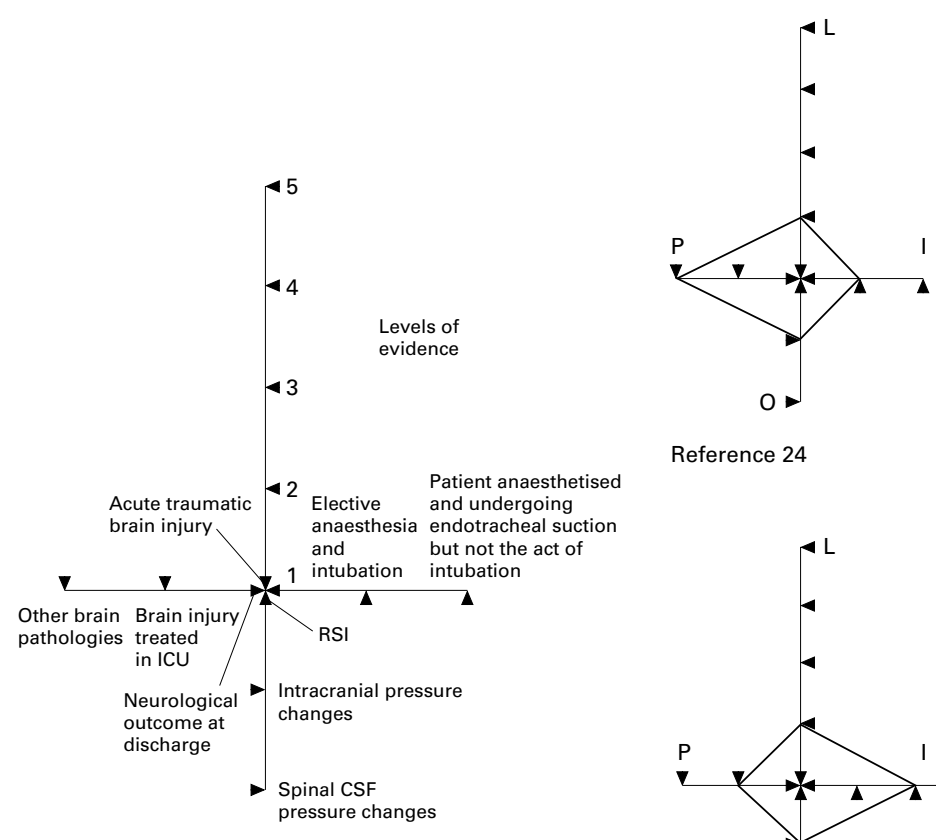

Reference 24

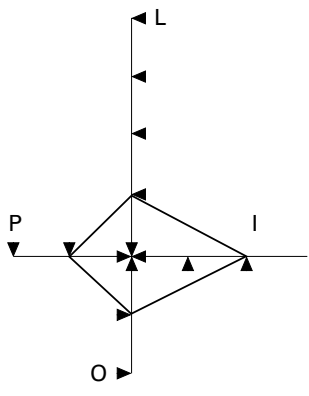

Reference 27 2a Systematic review of cohort studies

$2 b$ Individual cohort study including low quality RCT

3a Systematic review of case control studies

3 b Individual case control study

4 Case series and poor quality cohort/case control studies

5 Expert opinion with critical appraisal or based on physiology, bench research or first principles.

These levels formed the fourth axis.

\section{Papers identified}

A summary of each relevant paper is provided together with it's "grid" measurements.

1 Lidocaine prevents increased ICP after endotracheal intubation. Bedford et al. ${ }^{24}$ P2 I1 O1 L2

The patient group consisted of 20 patients undergoing elective neurosurgery for cerebral neoplasms. They all received intramuscular morphine, diazepam and atropine one hour prior to anaesthetic induction. Ten patients were given $1.5 \mathrm{mg} / \mathrm{kg}$ lidocaine IV "at the time of induction" and the other 10 received an identical volume of IV saline. "The medications were given in double-blind fashion and in random sequence"-the paper was no more specific about the randomisation process. All patients had a standardised induction with thiopentone, nitrous oxide/oxygen (50:50) and succinylcholine by continuous IV infusion. ICP changes were monitored for the five minutes after intubation. There was a significant blunting of the ICP rise, associated with intubation, in the group pretreated with lidocaine compared with saline pretreatment, $a$ treatment effect of $12 \mathrm{~mm} \mathrm{Hg}(\mathrm{p}<0.05)$. This commonly quoted paper involves a small
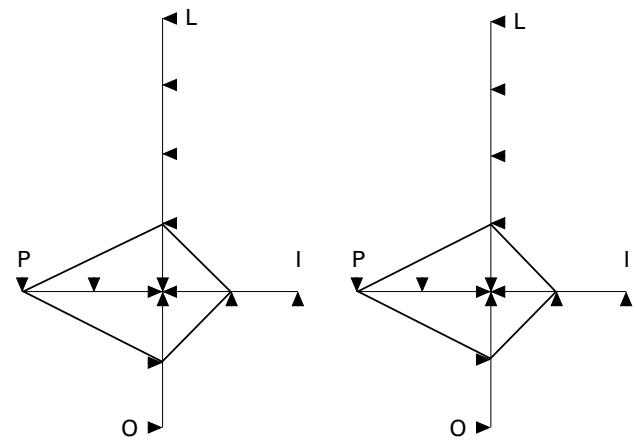

Reference 25

Reference 26

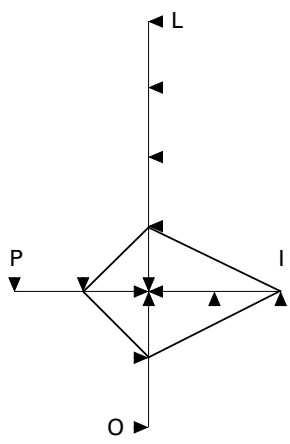

Reference 28

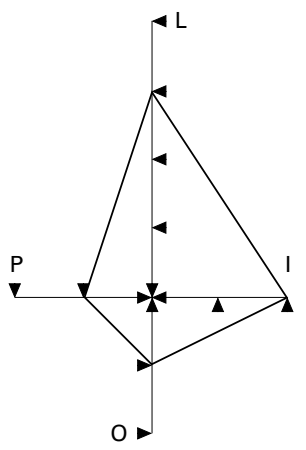

Reference 29 
number of patients without traumatic brain injury who are fully premedicated and not undergoing RSI. It does however suggest that lidocaine before treatment attenuated the rise in ICP associated with laryngoscopy and intubation when compared with placebo, in this setting.

2 Prevention of arterial pressure and intracranial pressure increase during endotracheal intubation in neurosurgery: esmolol versus lidocaine. Samaha et al. ${ }^{25}$ P2 I1 O1 L2

Twenty two patients undergoing elective neurosurgery for tumour resection or aneurysm clipping were randomly allocated, in double blind fashion, treatment with either lidocaine or esmolol both given IV after induction of anaesthesia with thiopentone, fentanyl, vecuronium and isoflurane, but 130 seconds before endotracheal intubation. ICP was measured directly and CPP calculated indirectly (MAP-ICP) in both groups. CPP decreased significantly after the administration of both study drugs, before intubation, but rose significantly in both groups after intubation, the average rise in both groups being $6 \mathrm{~mm} \mathrm{Hg}$. The authors concluded that both drugs do not completely prevent the rise in ICP and MAP associated with laryngoscopy and intubation. This study contained no control group to permit meaningful comparison of the results and the sample sizes were small. Neither the patient group nor the anaesthetic technique is relevant to our question.

3 Lidocaine before endotracheal intubation: intravenous or laryngotracheal? Hamill et al. ${ }^{26}$ P2 I1 O1 L2

Twenty two patients premedicated with morphine, diazepam and atropine, undergoing craniotomy and tumour resection of neoplasms greater than $3 \mathrm{~cm}$ in diameter were randomised to receive either intravenous or laryngotracheal lidocaine one minute after anaesthetic induction with thiopentone, succinylcholine and nitrous oxide/oxygen (50:50). After intubation $I C P$ rose by $6 \mathrm{~mm} \mathrm{Hg}$ in the IV group and $10 \mathrm{~mm}$ $\mathrm{Hg}$ in the laryngotracheal group. Neither group attenuated the haemodynamic responses to intubation. All the patients were receiving corticosteroid therapy preoperatively. Although the sample sizes are small this paper does suggest that pretreatment with IV lidocaine in this group of patients may be of benefit in attenuating the ICP rise associated with laryngoscopy and intubation. However, the results cannot be extrapolated to our very different patient group undergoing a quite different anaesthetic technique.

4 Intravenously administered lidocaine prevents intracranial hypertension during endotracheal suctioning. Donegan et al. ${ }^{27} \mathrm{P} 1 \mathrm{I} 2$ O1 L2

A randomised, double blind, crossover trial of 10 patients with closed head injuries ventilated on an intensive care unit. Each patient was receiving "moderate hyperventilation", mannitol and dexamethasone. In addition five of the patients were receiving pentobarbital hourly to lower abnormal ICPs. Each patient had either lidocaine or saline placebo two minutes beforeo endotracheal suctioning, the process then repeated on the same patient with the other study agent. ICP fell significantly, by $7 \mathrm{~mm} \mathrm{Hg}$, presuction after the administration of lidocaine when compared with saline. Endotracheal suctioning lead to a rise in the mean ICP after either agent, from 10 to 22 $\mathrm{mm} \mathrm{Hg}$ in the lidocaine group and 16 to $27 \mathrm{~mm}$ $\mathrm{Hg}$ in the saline group and it was the rise in this group which reached statistical significance. This paper has minor but important methodological weaknesses. Firstly, the small sample size reduces the power of the study and secondly the suction technique is not explicitly standardised in the paper. The conclusions drawn cannot be extrapolated to our patient group as the injuries were not acute and RSI was not a component of the study.

5 A randomised study of drugs for preventing increases in intracranial pressure during endotracheal suctioning. White et $a ._{.}{ }^{28} \mathrm{P} 1 \mathrm{I} 2$ O1 L2

An unblinded crossover study of 15 ventilated patients with diffuse brain injuries. Each patient was receiving mannitol, dexamethasone and had ventilation parameters similar to the paper by Donegan et al. All the patients received, in randomised sequence, one of 5 IV study agents (saline, fentanyl, thiopental, lidocaine and succinylcholine) or intratracheal lidocaine. Each patient received all treatments and these were repeated 2-8 times in differing sequences, with a minimum of one hour between each treatment. ICP and MAP values were recorded before, during and 3-5 minutes after "a standardised endotracheal suctioning procedure". The authors found that IV lidocaine and thiopental reduced the ICP pre suctioning by $4-6 \mathrm{~mm} \mathrm{Hg}$ but neither affected the ICP or MAP rise after it, the results mirroring those of the saline group. Interestingly, the most effective agent at attenuating the ICP rise was succinylcholine achieving statistical significance at the $p<0.01$ level. Succinylcholine had no effect on blunting the pressor response associated with endotracheal suctioning and so the authors recommend a combination of IV muscle relaxant and intratracheal lidocaine before carrying out this procedure. The results contradict those in the paper by Donegan et al. Again, in common with the other papers, these patients had non-acute injuries and were not undergoing RSI.

6 Effect of lidocaine on ICP response to endotracheal suctioning. Yano et al. ${ }^{29} \mathrm{P} 1 \mathrm{I} 2 \mathrm{O} 1$ L4

Nine patients with severe head injury were enrolled into this crossover cohort study. It is not clear from the paper if this work was done immediately after intubation in the emergency department or sometime later on the ICU. The method of intubation was not described. After intubation endotracheal suctioning was performed and the ICP measured. These measurements acted as the "controls". Each patient then received IV lidocaine $1,3,5,10$, and 15 minutes before endotracheal suctioning was performed. Six hours later intratracheal lidocaine was administered and the same protocol was performed. Intratracheal lidocaine was significantly more effective in suppressing ICP 
elevation caused by suctioning than IV lidocaine, a mean peak rise of $25 \mathrm{~mm} \mathrm{Hg}$ and $37 \mathrm{~mm}$ $\mathrm{Hg}$ respectively $(p<0.05)$, although neither method of lidocaine administration lowered the baseline ICP. This paper suffers from the fact that it is unrandomised, not placebo controlled, is unblinded and, again, the sample size is small.

\section{Conclusions}

The literature search revealed no paper that answered our question directly. The authors acknowledge that some potentially important papers may have been missed through the absence of hand searching of all possibly relevant journals but this was not possible and experts in this area were unable to identify any significant papers that were not evaluated.

The concept of "fit" depends on a number of assumptions that were made explicit. However, readers may for instance disagree with the values given to the different categories along each axis. For example, the difference along the outcome axis between neurological outcome and ICP may not be the same as the difference between ICP and CSF pressure. Readers could justifiably insert their own values.

The authors could find no evidence that in acute traumatic head injury pretreatment with IV lignocaine/lidocaine before a RSI reduces ICP or improves neurological outcome. The evidence for such an effect and the benefit of pretreatment comes from 42 fully premedicated patients undergoing elective neurosurgery, with elective anaesthesia not RSI, for tumour resection. The evidence obtained from studies looking at the ICP rise associated with endotracheal suctioning does not contribute to answering our question as it is not applicable to our patient population. The administration of IV lignocaine/lidocaine as a pretreatment in patients with acute head injury undergoing RSI should only occur in clinical trials.

$\mathrm{NR}$, who is the guarantor for this paper, undertook the literature search and appraisal and participated in writing the paper. MC initiated the study, was responsible for the graphical method and helped write the paper.

1 Pryse-Roberts C, Greene LT, Meloche R, et al. Studies of anaesthesia in relation to hypertension: II. Hemodynamic consequences of induction and endotracheal intubation. $\mathrm{Br}$ 7 Anaesth 1971;43:531-46.

2 Takeshima K, Noda K, Higaki M. Cardiovascular response to rapid anaesthesia induction and endotracheal intubation. Anaesth Analg 1964;43:201-8.

3 Millar Forbes A, Dally F. Acute hypertension during induction of anaesthesia and endotracheal intubation in normotensive man. Br $\mathcal{F}$ Anaesth 1970;42:618-24.

4 Mikawa K, Maekawa N, Goto R, et al. Prostaglandin E1 and tracheal intubation: Relationship between the cardiovascular responses and plasma catecholamine concentrations. f Int Med Res 1991;19:457-64.

5 Russell WJ, Morris RG, Frewin DB, et al. Changes in plasma catecholamine concentrations during endotracheal intubation. Br f Anaesth 1981;53:837-9.
6 Derbyshire DR, Chmielewski A, Fell D, et al. Plasma catecholamine response to tracheal intubation. $\mathrm{Br} \mathcal{F}$ Anaesth 1983;55:855-9.

7 Shribman AJ, Smith G, Achola J. Cardiovascular and catecholamine responses to laryngoscopy with and without tracheal intubation. Br F Anaesth 1987;50:295-9.

8 Tanaka K. The effects of intravenous injections of lidocaine on hemodynamics and catecholamine levels during endotracheal intubation in infants and children. Aichi-Gakuin f Dent Sci 1989;27:345-58.

9 Burney RG, Winn R. Increased cerebrospinal fluid pressure during laryngoscopy and intubation for induction of anaesthesia. Anaesth Analg 1975;54:687-9.

10 Rudy EB, Baun M, Stone K, et al. The relationship between endotracheal suctioning and changes in intracranial pressure: a review of the literature. Heart Lung 1986;15: 488-94.

11 Poulton TJ, James FM. Cough suppression by lidocaine. Anesthesiology 1979;50: 470-2.

12 Abou-Madi MN, Keszler H, Yacoub JM. Cardiovascular reactions to laryngoscopy and tracheal intubation following small and large intravenous doses of lidocaine. Can Anaesth Soc f 1977;24:12-19.

13 Splinter WM, Cervenko F. Haemodynamic responses to aryngoscopy and tracheal intubation in geriatric patients; effects of fentanyl, lidocaine and thiopentone. Can $\mathcal{F}$ Anaesth 1989;36:370-6.

14 Wilson IG, Meiklejohn BH, Smith G. Intravenous lignocaine and sympathoadrenal responses to laryngoscopy and intubation. The effect of varying the time of the injection. Anaesthesia 1991;46:177-80.

15 Kindler CH, Schumacher PG, Schneider MC, et al. Effects of intravenous lidocaine and/or esmolol on hemodynamic responses to laryngoscopy and intubation: A double-blind, controlled clinical trial. F Clin Anesth 1996;8:491-6.

16 Inada E, Cullen DJ, Nemeskal AR, et al. Effect of labetalol or lidocaine on the hemodynamic response to intubation: a controlled randomized double-blind study. F Clin Anesth 1989;1:207-13.

17 Miller CD, Warren SJ. I.V. Lignocaine fails to attenuate the cardiovascular response to laryngoscopy and tracheal intubation. Br f Anaesth 1990;65:216-19

18 Kobayashi TL, Watanabe K, Ito T. Lack of effect of i.v. lidocaine on cardiovascular responses to laryngoscopy and intubation. Masui 1995;44:579-82.

19 Chraemmer-Jorgensen B, Hoiland-Carlsen PF, Marving J, et al. Lack of effect of intravenous lidocaine on hemodynamic responses to rapid sequence induction of general anesthesia: a double-blind controlled clinical trial. Anesth Analg 1986;65:1037-41.

20 Allen RW, James MFM, Uys PC. Attenuation of the pressor response to tracheal intubation in hypertensive proteinuric pregnant patients by lignocaine, alfentanil and magnesium sulphate. Br F Anaesth 1991;66:216-23.

21 Silber SH. Rapid sequence intubation in adults with elevated intracranial pressure: a survey of emergency medicine residency programs. Am f Emerg Med 1997;15:263-7.

22 Butler JA, Clancy M, Robinson NA, et al. Survey of rapid sequence intubation in English emergency departments. EMF (in press).

23 Sackett DL, Richardson WS, Rosenberg W, et al. Evidence based medicine. How to practice and teach EBM. Edinburgh: Churchill Livingston, 2000.

24 Bedford RF, Winn HR, Tyson G, et al. Lidocaine prevents increased ICP after endotracheal intubation. In: Shulman $\mathrm{K}$, Mamorou A, Miller JD, et al, eds. Intracranial pressure IV. Berlin: Springer, 1980:595-8.

25 Samaha T, Ravussin P, Claquin C, et al. Prevention of arterial pressure and intracranial pressure increase during endotracheal intubation in neurosurgery: Esmolol versus lidocaine. Ann Fr Anesth Reanim 1996;15:36-40.

26 Hamill JF, Bedford RF, Weaver DC, et al. Lidocaine before endotracheal intubation: Intravenous or laryngotracheal? Anesthesiology 1981;55:578-81.

27 Donegan MF, Bedford RF. Intravenously administered lidocaine prevents intracranial hypertension during endotracheal suctioning. Anesthesiology 1980;52:516-18.

28 White PF, Schlobohm RM, Pitts LH, et al. A randomized study of drugs for preventing increases in intracranial pressure during endotracheal suctioning. Anesthesiology 1982; 57:242-4

29 Yano $\mathrm{M}$, Nishiyama $\mathrm{H}$, Yokota $\mathrm{H}$, et al. Effect of lidocaine on ICP response to endotracheal suctioning. Anesthesiology 1986;64:651-3 